\title{
Deferred Metabolism of Glucose by Clostridium tetanomorphum
}

\author{
By C. ANTHONY* AND J. R. GUEST \\ Department of Microbiology, University of Sheffield, Sheffield,
}

(Accepted for publication ro July 1968)

\begin{abstract}
SUMMARY
Clostridium tetanomorphum grew in medium containing yeast extract when supplied with glutamate, histidine, glucose, maltose or pyruvate. Measurements of $Q_{\mathrm{H}_{2}}$ values and specific activities for two enzymes, phosphofructokinase and $\beta$-methylaspartase indicated that the glucose and glutamate fermentations were inducible. With glucose, the onset of active glucose metabolism was delayed in preference for energy sources provided by yeast extract. The inhibitory mechanism which was responsible for this delay did not appear to be a typical enzyme repression but rather a catabolite inhibition. No significant changes in the cobalamin content of the organisms accompanied growth with different substrates.
\end{abstract}

\section{INTRODUCTION}

In a study of hydrogen evolution by anaerobes, washed suspensions of Clostridium tetanomorphum have been shown to ferment several amino acids and glucose, maltose, glycerol, pyruvate, fumarate, malate (Woods \& Clifton, 1938). The organism was used by Barker (1956) to elucidate the path of glutamate fermentation. This led to the discovery of the cobamide coenzymes which are synthesized by this organism, and which are essential coenzymes for glutamate mutase, the first enzyme in the glutamate fermentation (Barker, Weissbach \& Smyth, 1958). The object of the present work was to find some substrate other than glutamate which would serve as a major energy source, in order to study possible mechanisms of metabolic regulation in this strict anaerobe.

\section{METHODS}

The organism. Clostridium tetanomorphum (ATCC 3606) was maintained on slopes of blood agar (Oxoid) containing $7 \%$ horse blood and 0.1 M-sodium L-glutamate. After growth at $37^{\circ}$ for $24 \mathrm{hr}$ sealed with an alkaline pyrogallol plug, the cultures were stored at $2^{\circ}$ and remained viable for at least a month.

Media and growth of organisms. The salts + yeast extract basal medium of Barker, Smyth, Wilson \& Weissbach (1959) was modified by adding $\mathrm{NH}_{4} \mathrm{Cl}(0.02 \mathrm{M})$ and omitting sodium hydrosulphite; substrates (O.I M) were added after separate sterilization ( $12 \mathrm{I}^{\circ}$ for $\mathrm{I} 5 \mathrm{~min}$.). For the growth of small volumes ( $\mathrm{I} \circ \mathrm{ml}$.) $\perp$-tubes with alkaline pyrogallol plugs, sealed with bungs, were used; such tubes facilitated the measurement of growth when the organisms were beginning to sediment. The inoculum was I $\%(v / v)$ of a late exponential phase culture.

Two types of vessel were used for growth of the organism:

(I) For volumes of up to $200 \mathrm{ml}$., a $250 \mathrm{ml}$. Erlenmeyer flask with a $15 \times 125 \mathrm{~mm}$.

* Present address: Department of Physiology and Biochemistry, University of Southampton. 
test-tube welded at the base was used. The flask was sealed with an assembly which allowed tipping into the tube for measurement of growth and at the same time maintained an anaerobic atmosphere but prevented a build-up of gas pressure. This assembly consisted of another test-tube with a small hole at one side of the closed end; this was passed through a rubber bung and plugged with cottonwool. The flask + medium and the bung assembly were sterilized separately, appropriate substrates added, the medium inoculated and the bung put into position. The air space was evacuated, flushed with $\mathrm{H}_{2}+\mathrm{CO}_{2}(95+5, \mathrm{v} / \mathrm{v})$ and a plug soaked in alkaline pyrogallol was placed in the tube which was finally sealed with a bung fitted with a Bunsen valve (a piece of rubber tubing split diagonally across its width and closed with a glass rod at the distal end).

(2) When sampling was also required a flask, as described above (or larger), was fitted with an exit-tube which could be closed with silicone tubing and a screw clip. The neck of the flask was fitted with a bung which had several plugged tubes passing through it. One passed to the bottom of the flask for gassing with $\mathrm{H}_{2}+\mathrm{CO}_{2}(95+5$, $\mathrm{v} / \mathrm{v}$ ), a second was used to evacuate the gas space and a third passed to a Bunsen valve as described above. The freshly autoclaved medium was inoculated, the bung put into position and after evacuating, gas $\left(\mathrm{H}_{2}+\mathrm{CO}_{2}, 95+5, \mathrm{v} / \mathrm{v}\right)$ was bubbled through the medium for Io min. During incubation the bunsen valve was left open; for sampling it was closed with a screw clip, a positive pressure of gas $\left(\mathrm{H}_{2}+\mathrm{CO}_{2}\right)$ was introduced and a sample collected in an ice-cooled receptacle from the exit at the base of the flask. Because of the risks of contamination entailed by this procedure all cultures were tested for purity by streaking samples on blood agar (Oxoid) + glucose (O.I M) during each experiment. The samples withdrawn from the culture were cooled rapidly, the organisms sedimented by centrifuging ( $5000 \mathrm{~g}$ for $10 \mathrm{~min}$.) and the supernatant fluid retained for assay of substrates remaining in the medium.

Growth was assessed turbidimetrically with an EEL colorimeter (Evans Electroselenium Ltd.), with a green filter no. 624 and expressed as $\mathrm{mg}$. dry wt./ml., calculated from a standard curve.

Assay of pyruvate, glucose and glutamate. These compounds were assayed enzymically in dilutions of the culture supernatant fluids: pyruvate by the method of Bücher, Czok, Lamprecht \& Latko (1963), glucose by the method of Bergmeyer \& Bernt (I963) and glutamate according to Wyngaarden \& Ashton (I959).

Experiments with washed-cell suspensions. The fermentation of glucose, glutamate and pyruvate by suspensions of washed organisms was measured manometrically by using standard techniques. Organisms were harvested by centrifuging at $10,000 \mathrm{~g}$ at $0^{\circ}$ for $10 \mathrm{~min}$. washed twice in phosphate buffer $(0.05 \mathrm{M}, \mathrm{pH} \mathrm{7.4)}$ and resuspended in the same buffer. Hydrogen production by organisms (equiv. I0-20 mg. dry wt) was measured in phosphate buffer $(0.05 \mathrm{M}, \mathrm{pH} 7.0)$ with substrate $(0.05 \mathrm{M})$ in an atmosphere of $\mathrm{H}_{2}$ and with $\mathrm{KOH}(10 \%, \mathrm{w} / \mathrm{v})$ in the centre well.

Assay of enzymic activities. Cell-free extracts were prepared from washed organisms suspended in phosphate buffer $(0.02 \mathrm{M}, \mathrm{pH} 7.4)$ at a concentration equivalent to $300 \mathrm{mg}$. wet $\mathrm{wt} / \mathrm{ml}$. by disruption with an M.S.E. ultrasonic disintegrator for $20 \mathrm{sec}$. at $2^{\circ}$. The supernatant fluid obtained after centrifuging at $15,000 \mathrm{~g}$ for $20 \mathrm{~min}$. at $0^{\circ}$ was used for assaying phosphofructokinase by the method of Opie \& Newsholme (1967). The assay for $\beta$-methylaspartase was that of Barker et al. (1959) with the exception that tris buffer (O. I M, pH 9.0) was used. Protein was estimated by the method of Lowry, 
Rosebrough, Farr \& Randall (195I). Specific activities are expressed in terms of $\mu$ moles substrate changed/min./mg. protein.

Assay of cobalamin. Cobalamin was assayed microbiologically with Euglena gracilis var. bacillaris and Escherichia coli strain II3/3 (a cobalamin or methioninerequiring auxotroph; Davis \& Mingioli, 1950) using the methods described by Foster, Jones \& Woods (I96I). Clostridium tetanomorphum, harvested from $200 \mathrm{ml}$. medium when approaching maximum growth, was washed once with an equal volume of distilled water and resuspended in $10 \mathrm{ml}$. potassium phosphate buffer $(0.02 \mathrm{M}, \mathrm{pH} 6.5)$ containing $\mathrm{KCN}$ (0.0I M). Samples of this were used for protein estimations and for cobalamin assay. For the latter, samples $\left(3 \mathrm{ml}\right.$.) were autoclaved at $115^{\circ}$ for $10 \mathrm{~min}$., cooled and digested with Seitz-filtered papain $\left(0.3 \mathrm{mg}\right.$. $/ \mathrm{ml}$. final concn.) at $25^{\circ}$ for $48 \mathrm{hr}$. The samples were then adjusted to $\mathrm{pH} 4.5$ with acetic acid, heated to $100^{\circ}$ for Io min., and denatured protein removed by centrifuging. The supernatant fluid was used for the assay of cobalamin with cyanocobalamin (vitamin $B_{12}$ ) as standard. Initially the medium contained $18 \mathrm{~m} \mu \mathrm{g}$. vitamin $B_{12} / \mathrm{ml}$. for Euglena assay and after growth the cultures contained approximately $45 \mathrm{~m} \mu \mathrm{g}$. vitamin $\mathrm{B}_{12} / \mathrm{ml}$, , more than $80 \%$ of which was recovered in the washed organisms.

Materials. The enzymes required for glucose, glutamate and pyruvate assays were purchased from C. F. Boehringer und Soehne G.m.b.H. Mannheim, Germany, as were NAD, $\mathrm{NADH}_{2}, \mathrm{ATP}$ and glucose-6-phosphate. Twice-crystallized papain and cyanocobalamin were from British Drug Houses Ltd., Poole, Dorset. The sources of other reagents were: 3-acetylpyridine NAD (Calbiochem, Los Angeles, Calif., U.S.A.), DL-citramalic acid (Aldrich, Milwaukee, Wisconsin, U.S.A.), mesaconic acid (Fluka A.-G., Buchs SG., Switzerland), $\beta$-methyl-DL-aspartate (Sigma Chemical Co., St Louis, Ma., U.S.A.) and yeast extract (Difco Laboratories Inc., Detroit I, Michigan, U.S.A.).

\section{RESULTS}

\section{Growth experiments}

When Clostridium tetanomorphum was incubated in media containing yeast extract $(0.3 \%)$ growth occurred with the following substrates: glucose, maltose, L-glutamate, L-histidine and to a lesser extent with pyruvate. No growth was observed with the yeast extract alone, nor with added fructose, L-aspartate, L-threonine, $\alpha$-ketoglutarate, succinate, fumarate or L-malate, some of which were originally shown to be fermented by washed suspensions of the organism (Woods \& Clifton, 1938). $\beta$-Methyl-DLaspartate, mesaconate and DL-citramalate, intermediates in the fermentation of glutamate, were also unable to support the growth of the organism in this medium. With glutamate $(0.1 \mathrm{M})$ as substrate maximum growth was obtained when the concentration of yeast extract was increased to about $\mathrm{r} \cdot 5 \%$ (Fig. I) and this concentration was used in all subsequent experiments. Even at this concentration of yeast extract, growth in the absence of glutamate was less than $5 \%$ of the amount reached in its presence.

Growth of Clostridium tetanomorphum with glutamate, glucose or pyruvate became linear after a short exponential phase. The mean generation times calculated from these short phases were: for glutamate, $\mathrm{I} \cdot 2 \mathrm{hr}$; glucose $\mathrm{I} \cdot 5 \mathrm{hr}$; pyruvate, $\mathrm{I} \cdot 2 \mathrm{hr}$. Measurements of growth yields were made to ensure that glucose and pyruvate were serving as principal energy sources (Fig. 2). In the region, where the yields were pro- 
portional to substrate concentration, no substrate remained in the medium after growth and the yield coefficients calculated were $4 \mathrm{I} \cdot 0 \mathrm{~g}$. dry wt organism/mole of glucose consumed, and 6.0 and 8.0 for glutamate and pyruvate, respectively. Assuming that $Y_{A T P}$ is Io then the corresponding ATP yields, 4.I for glucose, 0.6 for glutamate and 0.8 for pyruvate, are consistent with the anticipated yields of 0.5 to $1 \cdot 0$ mole ATP/mole glutamate or pyruvate converted to butyrate or acetate, respectively, by the recognized metabolic routes, plus an extra 2 moles ATP/mole of glucose converted to pyruvate via the glycolytic pathway.

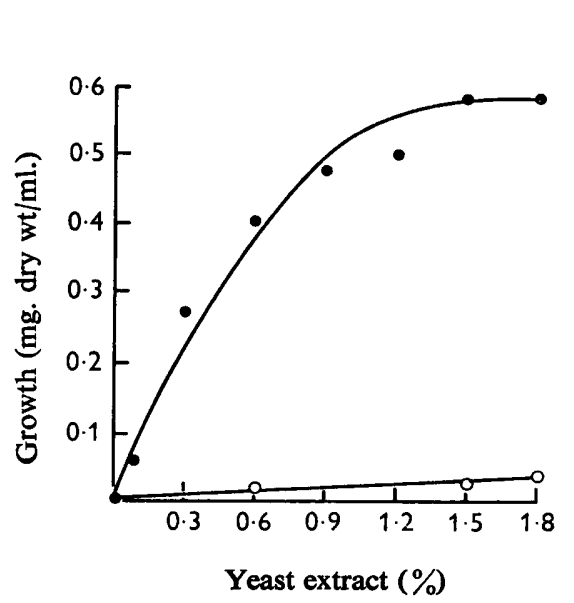

Fig. I

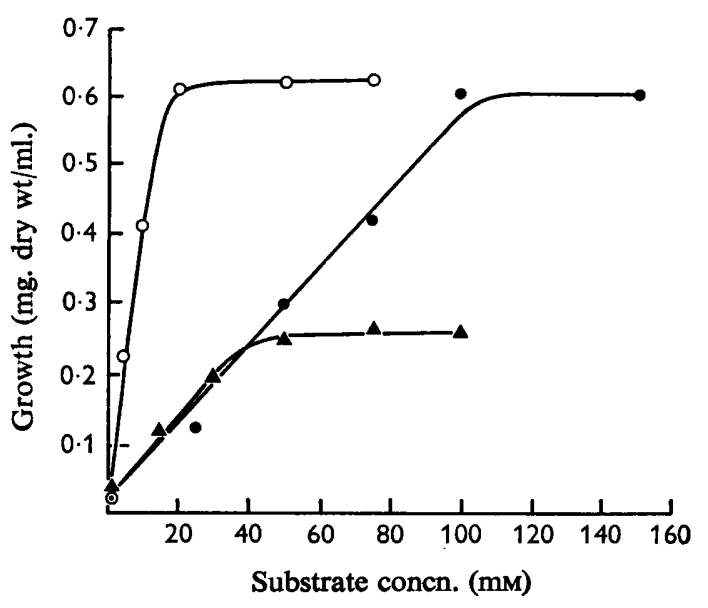

Fig. 2

Fig. I. The effect of yeast extract concentration $(\%, w / v)$ on the growth of Clostridium tetanomorphum. $\bullet$, With glutamate (O.I M); $\mathrm{O}$, without glutamate.

Fig. 2. Growth as a function of substrate concentration. The medium contained yeast extract $(\mathbf{I} \cdot 5 \%$. 0 , Glutamate; $O$, glucose; $\boldsymbol{\Delta}$, pyruvate.

Growth with mixed substrates. During growth on mixtures of glutamate and glucose higher growth yields were obtained than with either substrate alone. It was of interest to determine whether the organisms exhibited a preference for one or other of the substrates under these conditions. The growth curves for Clostridium tetanomorphum grown on mixtures of glucose and glutamate occasionally exhibited a diauxic phase; more often a short exponential phase was followed by a phase of slower growth. Measurements of the concentrations of substrates in the medium at different times indicated that glutamate was metabolized first and that only when the glutamate had disappeared was there any significant decrease in the glucose concentration (Fig. 3). The same results were obtained whether the inoculum was derived from organisms grown with glucose or from glutamate.

Increasing the glutamate concentration relative to glucose had the effect of delaying further the onset of glucose utilization (Fig. 4). However, it appeared that glutamate was not solely responsible for this effect, because glucose was still fermented at a later stage in growth even though considerable concentrations of glutamate remained in the medium. Increasing the concentration of yeast extract had the same effect on the onset of glucose metabolism as did increased glutamate (Fig. 4). Thus it was realized that the yeast extract was serving as an energy source and direct measurement of the 
utilization of glucose by organisms growing with yeast extract + glucose (but without glutamate) confirmed this: glucose was not used appreciably until about a quarter of the growth had occurred. No effect on the utilization of glutamate of doubling the yeast extract concentration was detected.

So, contrary to what might have been expected from the growth yield results, glucose was not the principal energy source during the initial period of growth, but rather it appeared to be fulfilling the role of an essential metabolite. Presumably, the amino acids in the yeast extract served as preferential energy sources. This implied

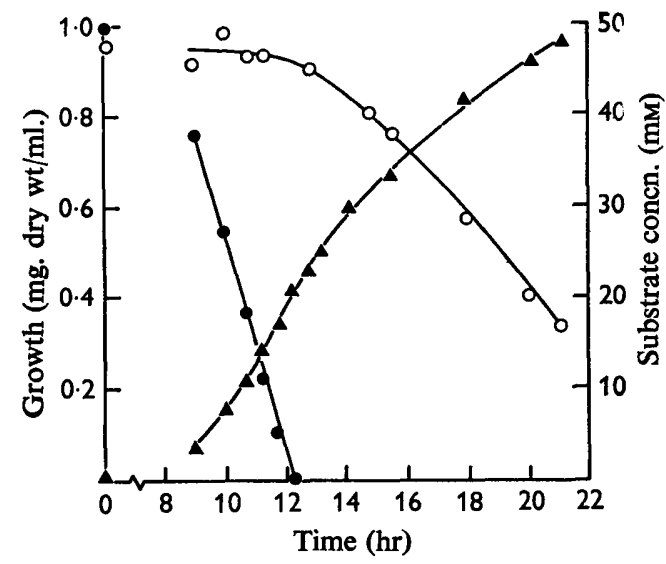

Fig. 3

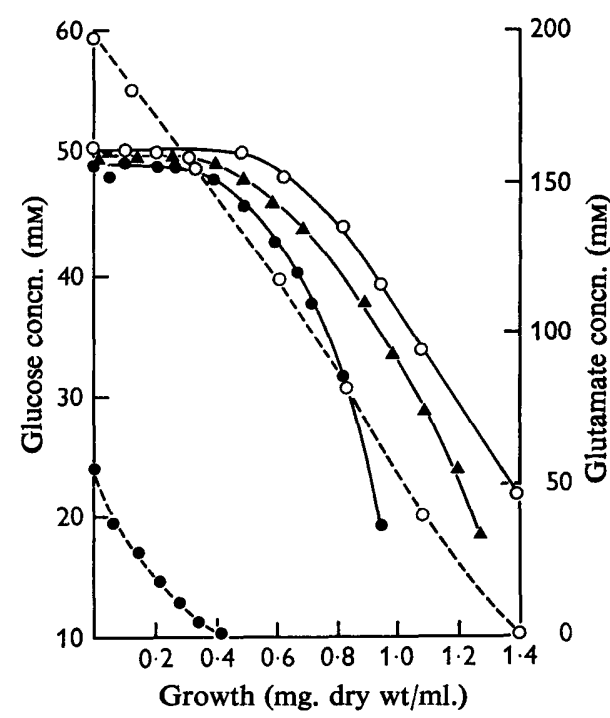

Fig. 4

Fig. 3. Growth of Clostridium tetanomorphum on a mixture of glutamate and glucose. The medium contained yeast extract $(1.5 \%)$ and the inoculum was $0 . I \%(v / v)$ of a culture of organisms grown with glutamate. Concentrations of substrates remaining were: $\bullet$, glutamate; $O$, glucose. $\boldsymbol{\Delta}$, Growth.

Fig. 4. The effect of glutamate and yeast extract concentrations on the utilization of glucose during growth of Clostridium tetanomorphum. Substrate concn.: - _, glucose; - - , glutamate. O, Glutamate initial concn., $0.05 \mathrm{M}$, yeast extract, $\mathrm{I} .5 \%$; 0 , glutamate initially $0.2 \mathrm{M}$, yeast estract, $\mathrm{I} \cdot 5 \% ; \boldsymbol{\Delta}$, glutamate initially $0.05 \mathrm{M}$, yeast extract $3.0 \%$. The initial concn. of glucose was $0.05 \mathrm{M}$ in all cases and the inoculum was $0.1 \%(\mathrm{v} / \mathrm{v})$ of a glutamate grown culture.

that the mean generation time for growth with glucose, calculated during the early log phase, was not truly representative of the value for growth with glucose as the energy source. During growth with glutamate or pyruvate, the substrate was used from the medium at the rate expected from the growth yield results. Furthermore, with a mixture of glutamate and pyruvate, there was no increase in growth yield and both substrates were used simultaneously.

\section{Induction of glucose and glutamate fermentations}

A possible explanation for delayed glucose fermentation and preferential metabolism of yeast extract components is that the enzymes of the glucose pathway are inducible and that their formation is repressed by a constituent of the yeast extract or 
by a product of its metabolism; derepression would then accompany depletion of the constituents from the medium. This would then be an example of catabolite repression (Magasanik, 196r). Alternatively, one or more steps in the path of glucose utilization might be inhibited by constituents or catabolites of the yeast extract; depletion of such compounds during the early phase of growth would annul the inhibition and permit glucose utilization.

To investigate these possibilities washed suspensions of organisms grown under different conditions were tested for ability to ferment glucose, glutamate or pyruvate. The rate of hydrogen evolution with these substrates under anaerobic conditions $\left(Q_{\mathrm{H}_{2}}\right)$, was taken as an index of their fermentative capacity. Suspensions of glucosegrown organisms produced hydrogen with glucose or pyruvate but not with glutamate; conversely, glutamate-grown organisms produced hydrogen with glutamate or pyruvate but not with glucose (Table I). Pyruvate-grown organisms produced significant quantities of hydrogen only when incubated with pyruvate. The specific activity of a key enzyme of the Embden-Meyerhof pathway, phosphofructokinase, in extracts of organisms grown with glucose was four times greater than for organisms grown with glutamate, and the specific activity of $\beta$-methylaspartase, the second enzyme in the glutamate pathway, was about 12 times greater in extracts of glutamate-grown organisms (Table I). Thus it appears that both the glucose and glutamate fermentations are inducible in Clostridium tetanomorphum.

\section{Table I. Properties of organisms grown with different substrates}

Organisms were grown in medium containing substrate ( $(0 \cdot \mathrm{I} M)$ and yeast extract $(\mathrm{I} \cdot 5 \%)$. $Q_{\mathbf{H}_{2}}$ and enzyme specific activities were measured as described in the Methods section.

\begin{tabular}{lccc} 
& \multicolumn{3}{c}{ Growth substrate } \\
\cline { 2 - 4 }$Q_{\mathrm{H}_{2}}$-glutamate & Glutamate & Glucose & Pyruvate \\
$Q_{\mathrm{H}_{2}}$-glucose & 44 & 0 & 0 \\
$Q_{\mathrm{H}_{2}}$-pyruvate & 0 & 15 & 0 \\
Phosphofructokinase & 24 & 20 & 45 \\
$\beta$-Methylaspartase & 0.036 & 0.150 & -
\end{tabular}

To determine whether the delay in glucose utilization was due to repression of the enzymes for glycolysis, the $Q_{\mathrm{H}_{2}}$ for glucose was measured for organisms withdrawn from cultures which had glucose present from the time of inoculation (Fig. 5). Glutamate-grown organisms were used as inoculum and following an early period of induction, during which less than $10 \%$ of growth occurred, the $Q_{\mathrm{H}_{2}}$ for glucose remained more or less constant and there was no significant increase during the period in which glucose was being fermented at an increased rate. However, when glucose was added to a culture which had just exhausted a growth-limiting amount of glutamate, the $Q_{\mathrm{H}_{2}}$ for glucose increased from 0 to I3 (Fig. 6) and values of 7 and 8 were reached before the glucose concentration started to fall. Here again, the growth began before glucose was "tilized. Concomitant with the induction of ability to ferment glucose there was an increase in the specific activity of phosphofructokinase (Fig. 6). These observations confirm that the glucose fermentation was inducible and, in addition, they indicate that control of glucose fermentation was achieved by a mechanism 
other than enzyme repression, because even when the fermentation enzymes were present glucose was not utilized from the medium.

In similar experiments with organisms growing on glutamate +glucose from the time of inoculation, the $Q_{\mathrm{H}_{2}}$ for glutamate and the specific activity of $\beta$-methylaspartase were almost halved between the time of glutamate disappearance and the termination of growth. Such changes were consistent with a cessation of further synthesis of enzymes of the glutamate pathway during the period in which the growth yield doubled. Furthermore, when glutamate was added to a culture of Clostridium tetanomorphum growing with glucose, the rate of glutamate utilization increased rapidly

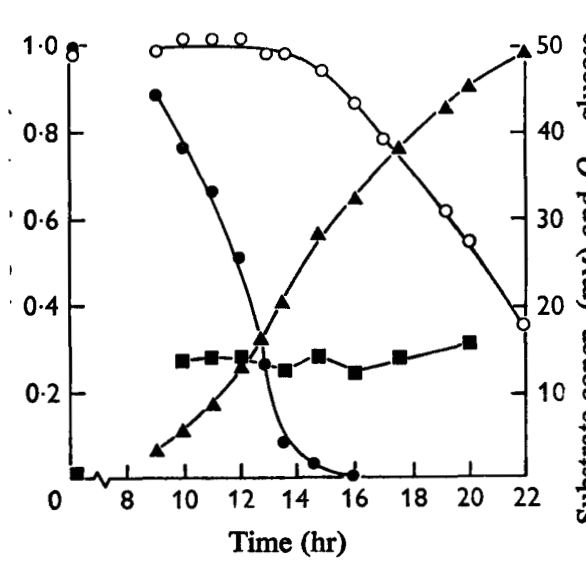

Fig. 5

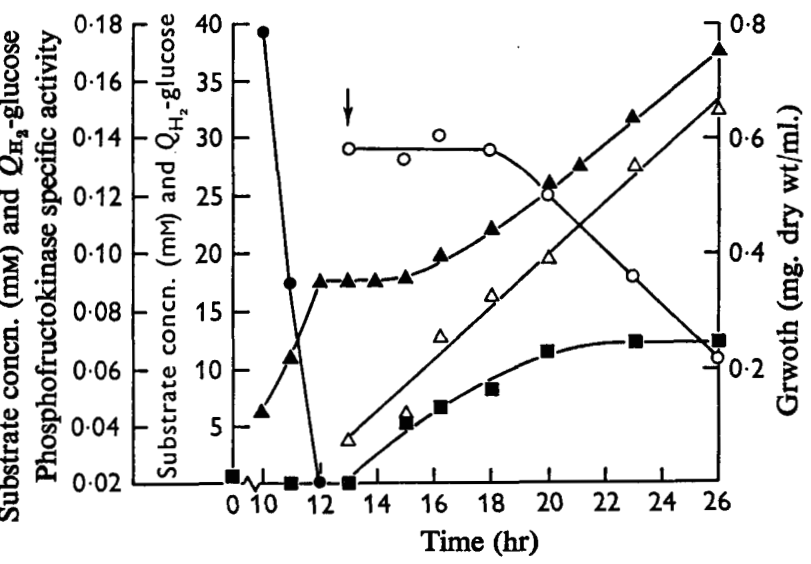

Fig. 6

Fig. 5. The capacity to ferment glucose of organisms harvested at various phases of growth from a medium containing yeast extract $(1.5 \%)$ and a mixture of glutamate and glucose starting with an inoculum $(0.1 \%, v / v)$ grown with glutamate. $\Delta$, Growth; $\Theta$, glutamate concn.; O, glucose concn.; $\boldsymbol{\square}, \boldsymbol{Q}_{\mathrm{H}_{\mathbf{2}}}$ for glucose.

Fig. 6. Induction of the ability to ferment glucose ( $Q_{\mathrm{H}_{2}}-$ glucose), and of phosphofructokinase after the addition of glucose $(28 \mathrm{~mm}$ at $13 \mathrm{hr}$ indicated by the arrow) to organisms grown with glutamate (60 mM). $\Delta$, Growth;, glutamate concn.; $O$, glucose concn.; $\triangle$, specific activity of phosphofructokinase; $\boldsymbol{\square}, Q_{\mathrm{H}_{\mathbf{2}}}$ for glucose.

and there was a marked increase in growth rate. Simultaneously, there was a progressive increase in the $Q_{\mathrm{H}_{2}}$ value for glutamate of samples removed from the culture after increasing periods of time.

Cobalamin content of Clostridium tetanomorphum. In view of the inducible nature of the glutamate fermentation it was of interest to determine whether the cobalamin content of the organisms was regulated in a co-ordinate fashion, because of the participation of cobalamin in the metabolism of glutamate. No marked differences in cobalamin content were observed for organisms grown with different substrates (Table 2). Even when the glutamate content of the glucose medium was decreased from the usual $5 \mathrm{~mm}$ to about $0.5 \mathrm{~mm}$ by previous treatment of yeast extract with glutamate decarboxylase, there was only a slight decrease in the cobalamin content. The values obtained with Euglena gracilis assay were about 20 times higher than those with the Escherichia coli $113 / 3$ assay. This discrepancy was presumably because the organism synthesized predominantly the coenzyme form of pseudo-vitamin $B_{12}$ and although 
pseudo-vitamin $\mathrm{B}_{12}$ and cyanocobalamin (vitamin $\mathrm{B}_{12}$ ) are equally active for $E$. gracilis previous reports indicate that pseudo-vitamin $B_{12}$ is only one-tenth as active for Escherichia coli $113 / 3$ (Smith, 1965).

Table 2. Cobalamin content of Clostridium tetanomorphum harvested from different media

Organisms were grown in media containing $\mathrm{I} \cdot 5 \%$ yeast extract and the substrate indicated (0.1 M). Assay of cobalamin was as described in the Methods section.

\begin{tabular}{|c|c|c|}
\hline \multirow[b]{2}{*}{ Substrate } & \multicolumn{2}{|c|}{$\begin{array}{l}\text { Cobalamin content } \\
(\mu \mathrm{mg} \cdot / \mathrm{mg} \cdot \text { protein })\end{array}$} \\
\hline & $\begin{array}{l}\text { Euglena } \\
\text { gracilis }\end{array}$ & $\begin{array}{c}\text { Escherichia } \\
\text { coli } \mathrm{I}_{3} / 3\end{array}$ \\
\hline Glutamate & 256 & I $2 \cdot I$ \\
\hline Histidine & 274 & 12.9 \\
\hline Pyruvate & 326 & $14 \cdot 8$ \\
\hline Glucose & 245 & $13 \cdot 6$ \\
\hline Glucose* & 190 & $10 \cdot 2$ \\
\hline Maltose & 234 & $8 \cdot 4$ \\
\hline
\end{tabular}

* The yeast extract used for preparing the medium was treated with glutamate decarboxylase to remove glutamate.

\section{DISCUSSION}

Until recently very little was known about the control of metabolic processes in strict anaerobes, and it appeared that Clostridium tetanomorphum would provide a suitable subject for such a study. However, contrary to preliminary indications that single substrates would serve as principal energy source, it is now clear that this organism has considerable nutritional complexity. The apparent discrepancy between the observation that the final growth yield may be proportional to the initial glucose concentration, and the observation that the extent of glucose utilization at a given time during growth is not proportional to the amount of growth, makes it difficult to be certain at any time whether a substrate is functioning as a principal energy source. The importance of the yeast extract as an energy source cannot be overlooked. Furthermore, when growth has ceased for reasons other than lack of energy sources, it is possible that an uncoupled metabolism of substrate may occur.

Glutamate, pyruvate and glucose all promoted the growth of Clostridium tetanomorphum in the yeast extract medium. However, glutamate and pyruvate were metabolized without appreciable delay, whereas glucose utilization was deferred. The delay in glucose catabolism suggests that glucose promotes growth primarily by serving as a carbon source or growth factor and that the organism possesses a regulatory mechanism for glucose conservation. The role of glucose as energy source was of secondary importance during the early part of growth since components of the yeast extract were used preferentially for this purpose. In addition to glutamate and histidine (which permit growth), the energy-yielding components of the yeast extract undoubtedly include aspartate, serine, cysteine, methionine and tyrosine, which are known to be fermented by washed suspensions of this organism (Woods \& Clifton, 1938). Rapid metabolism of these compounds via pyruvate would make a significant contribution to the amount of energy available during the initial stages of growth, when growth is not prevented by lack of a suitable carbon source. By contrast with glucose the early 
metabolism of glutamate and pyruvate suggests that these are preferred energy sources which may promote growth by sparing the carbon sources or essential metabolites in yeast extract. The absence of growth with yeast extract alone could thus be interpreted as due to a deficiency of carbon sources, or to a lack of sufficient energy sources.

In yeast the glycolytic enzymes are induced by increasing the glucose concentration in the medium (Hommes, 1966) and, Lee \& Ordal (1967) have shown that Clostridium thermosaccharolyticum has increased amounts of glycolytic enzymes but a lower rate of pyruvate fermentation when grown with glucose as compared, with pyruvate. In the present work with $C$. tetanomorphum both the glycolytic and the glutamate pathways were inducible and the capacity to ferment pyruvate was somewhat lower in organisms grown with glutamate or glucose as compared with the capacity in organisms grown with pyruvate. That pyruvate is fermented by glutamate or glucose-grown organisms is to be expected since 'phosphoroclastic' metabolism of pyruvate is thought to be common to glutamate, glucose and pyruvate fermentations.

No evidence has been obtained here for the existence of a repression mechanism for the glutamate pathway of Clostridium tetanomorphum. Glutamate metabolism appears to be unaffected by the presence of pyruvate, glucose or increased concentrations of yeast extract. Similarly, the results indicate that the regulation of glucose metabolism is not due to catabolite repression by glutamate or some component of the yeast extract, because the capacity to ferment glucose was induced even in their presence. Nevertheless, the onset of glucose metabolism was deferred in organisms possessing the capacity to ferment glucose, so it appears that the control mechanism resides in the inhibition of one or more enzymes of glycolysis by a metabolizable constituent of the medium or a product thereof.

Many mechanisms might be suggested to account for this feed-back or catabolite inhibition. It seems unlikely that pyruvate is directly responsible for the inhibition because tests have shown that there was no significant increase in the concentration of pyruvate during the period of deferred glucose metabolism. Nor could glutamate be solely responsible because glucose metabolism was initiated in the presence of excess glutamate. Some component of the yeast extract might have a direct inhibitory effect on one of the enzymes of glycolysis or on a glucose permease. However, a more likely explanation is that glycolysis in Clostridium tetanomorphum is regulated by a feed-back inhibition of one or more of the glycolytic enzymes by a catabolite of a yeast component. Such feed-back mechanisms have been found in other micro-organisms and in higher organisms. Possible examples are the inhibition of phosphofructokinase by ATP and citrate (Passonneau \& Lowry, 1964; Lowry \& Passonneau, 1964) and of pyruvatekinase and gluconokinase by acetylCoA (Weber, Lee \& Stamm, 1967). Also in this context a delay in the removal of glucose from the medium by Escherichia coli has been thought to be due to a product of pyruvate metabolism (Morgan \& Kornberg, 1967). The absence of any marked variation in the cobalamin content of C. tetanomorphum grown in different media suggests that synthesis of the vitamin is not co-ordinated with the fermentation of glutamate. A regulatory mechanism may involve an influence of glutamate on the conversion of cobamides to the coenzyme forms, or alternatively the vitamin may have as yet unknown functions in the metabolism, for example of other amino acids, and enzymes for its synthesis may therefore always be induced in the medium used here. 
We are indebted to $\mathrm{Mr}$ R. Bacon for technical assistance. The work was aided by grant B/SR/3432 from the Science Research Council.

\section{REFERENCES}

BARXER, H. A. (1956). Bacterial Fermentations. New York: John Wiley and Sons, Inc.

Barker, H. A., Weissbach, H. \& SMYth, R. D. (1958). A coenzyme containing pseudovitamin $B_{12}$. Proc. natn. Acad. Sci. U.S.A. 44, 1093.

Barker, H. A., SmYTh, R. D., Wilson, R. M. \& Weissbach, H. (1959). The purification and properties of $\beta$-methylaspartase. J. biol. Chem. 234, 320.

BERGMEYER, H. \& BeRNT, E. (1963). In Methods of Enzymatic Analysis. Ed. by H. Bergmeyer, p. 23. Verlag Chemie Gmb H.

Bücher, T., CZOK, R., LAMPrecht, W. \& LATKo, E. (I963). In Methods of Enzymatic Analysis, Ed. by H. Bergmeyer, p. 253. Verlag Chemie Gmb H.

Davis, B. D., \& Mingrolr, E. S. (1950). Mutants of Escherichia coli requiring methionine or vitamin $B_{12}$. J. Bact. 60, 17.

Foster, M. A., Jones, K. M. \& Woods, D. D. (196I). The purification and properties of a factor containing vitamin $\mathrm{B}_{12}$ concerned in the synthesis of methionine by Escherichia coli. Biochem. J. 80, 519.

Hommes, F. A., (1966). Effect of glucose on the level of glycolytic enzymes in yeast. Archs Biochem. Biophys. xI4, 231.

LEE, C. K. \& ORDAL, Z. J. (1967). Regulatory effect of pyruvate on the glucose metabolism of Clostridium thermosaccharolyticum. J. Bact. 94, 1530.

Lowry, O. H. \& PassonneaU, J. V. (1964). A comparison of the kinetic properties of phosphofructokinase from bacterial, plant and animal sources. Arch. exp. Path. Pharmak. 248, 185.

Lowry, O. H., Rosebrough, N. J., FARR, A. L. \& Randall, R. J. (I95I). Protein measurement with the Folin phenol reagent. J. biol. Chem. 193, 265.

MagasantK, B. (1961). Catabolite repression. Cold Spring Harb. Symp. quant. Biol. 26, 249.

MoRgAN, M. J. \& KorNBERG, H. L. (1967). Effect of pyruvate on hexose metabolism by Escherichia coli. Biochem. J. 103, 57P.

OpIE, L. H. \& Newsholme, E. A. (1967). The activities of fructose-1,6-diphosphatase, phosphofructokinase and phosphoenolpyruvate carboxykinase in white muscle and red muscle. Biochem.J. I03, 39I.

PassonneaU, J. V. \& LowRY, O. H. (1964). The role of phosphofructokinase in metabolic regulation. In Advances in Enzyme Regulation. Ed. by G. Weber, 2, 265. London: Macmillan.

SMrTH, E. L. (1965). Vitamin $B_{12}$. London: Methuen.

Weber, G., LeE, M. A. \& StAmM, W. B. (1967). Inhibition of pyruvate kinase and glucokinase by acetylCoA and inhibition of glucokinase by phosphoenolpyruvate. Life Sciences 6, 244I.

Woods, D. D. \& Clifton, C. E. (1938). Studies in the metabolism of the strict anaerobes (genus Clostridium). VI. Hydrogen production and amino acid utilization by Clostridium tetanomorphum. Biochem. J. 3I, 1774 .

WyngaARden, J. B. \& Ashton, D. M. (1959). The regulation of activity of phosphoribosylpyrophosphate amidotransferase by purine ribonucleotides; a potential feedback control of purine biosynthesis. J. biol. Chem. 234, I492. 